\title{
A Parameter Free Iterative Method for Solving Projected Generalized Lyapunov Equations ${ }^{1}$
}

\author{
Yiqin Lin ${ }^{\mathrm{a}}$, Liping Zhou ${ }^{\mathrm{b}}$, Liang Bao \\ ${ }^{a, b}$ Department of Mathematics and Computational Science, Hunan University of of Science and Engineering, \\ Yongzhou 425100, P. R. China \\ ${ }^{c}$ Department of Mathematics, East China University of Science and Technology, Shanghai, 200237, P. R.
}

China

\begin{abstract}
This paper is devoted to the numerical solution of projected generalized continuous-time Lyapunov equations with low-rank right-hand sides. Such equations arise in stability analysis and control problems for descriptor systems including model reduction based on balanced truncation. A parameter free iterative method is proposed. This method is based upon a combination of an approximate power method and a generalized ADI method. Numerical experiments presented in this paper show the effectiveness of the proposed method.
\end{abstract}

Index Terms: Projected generalized Lyapunov equation; ADI method; Parameter free method; C-stable

(C) 2012 Published by MECS Publisher. Selection and/or peer review under responsibility of the Research Association of Modern Education and Computer Science.

\section{Introduction}

In this paper we consider the projected generalized continuous-time Lyapunov equation

$$
\left\{\begin{array}{l}
E X A^{T}+A X E^{T}+P_{l} B B^{T} P_{l}^{T}=0, \\
X=P_{r} X P_{r}^{T}
\end{array}\right.
$$

\footnotetext{
${ }^{1}$ This work is partially supported by NSFC Grant \#10801048 to Y. Lin and NSFC Grant \#10926150 to L. Bao.

* Corresponding author.

E-mail address: ${ }^{\mathrm{a} y q l i n @ y a h o o . c n ; ~}{ }^{\mathrm{b}}$ lpzhou_huse@yahoo.cn; ${ }^{\mathrm{c}}$ nlbao@yahoo.cn
} 
Where $A, E \in R^{n \times n}, B \in R^{n \times m}$, and $X \in R^{n \times n}$ is the sought-after solution. Here, $P_{l}$ and $P_{r}$ are the spectral projectors onto the left and right deflating subspaces corresponding to the finite eigenvalues of the pencil $\lambda E-A$, respectively. It has been shown in [1] that if the pencil $\lambda E-A$ is c-stable, i.e., all its finite eigenvalues have negative real part, then the projected generalized continuous-time Lyapunov equation (1) has a unique, symmetric and positive semidefinite solution $X$.

We assume that the pencil $\lambda E-A$ is regular, i.e., $\operatorname{det}(\lambda E-A)$ is not identically zero. Under this assumption, the pencil $\lambda E-A$ has the Weierstrass canonical form [2]: there exist nonsingular $n \times n$ matrices $W$ and $T$ such that

$$
E=W\left[\begin{array}{ll}
I & 0 \\
0 & N
\end{array}\right] T, \quad A=W\left[\begin{array}{ll}
J & 0 \\
0 & I
\end{array}\right] T
$$

Where $J$ and $N$ are block diagonal matrices with each diagonal block being a Jordan block. The eigenvalues of $J$ are the finite eigenvalues of the pencil $\lambda E-A$ and $N$ corresponds to the eigenvalue at infinity. Using (2), $P_{l}$ and $P_{r}$ can be expressed as

$$
P_{l}=W\left[\begin{array}{ll}
I & 0 \\
0 & 0
\end{array}\right] W^{-1}, \quad P_{r}=T^{-1}\left[\begin{array}{ll}
I & 0 \\
0 & 0
\end{array}\right] T .
$$

The projected generalized continuous-time Lyapunov equation (1) arises in stability analysis and control design problems for descriptor systems including the characterization of controllability and observability properties, balanced truncation model order reduction, determining the minimal and balanced realizations as well as computing $\mathrm{H}_{2}$ and Hankel norms; see [3,4] and the references therein.

Several numerical methods have been proposed in the literature for solving the projected generalized Lyapunov equation (1). In [5], two direct methods, the generalized Bartels-Stewart method and the generalized Hammarling method, were proposed for the projected generalized Lyapunov equation of small or medium size. Iterative methods to solve large sparse projected generalized Lyapunov equations have also been proposed. Stykel [6] extended the ADI method and the Smith method to the projected equation. Moreover, low-rank versions of these methods were also presented, which could be used to compute low-rank approximations to the solution.

The ADI method requires to select shift parameters. To obtain optimal shift parameters, we need to solve a rational min-max problem. This problem is only solved for standard Lyapunov equations with symmetric coefficient matrices. For the non-symmetric case, some heuristic shift selection procedures have been proposed to compute the suboptimal ADI shift parameters, see [7,8]. However, these shift selection procedures do not work well for some applications. If some poor shift parameters are provided by the shift selection procedure, it can lead to very slow convergence in the ADI method.

Recently, a parameter free method was proposed in [9] for solving the large-scale standard Lyapunov equation in low-rank factored form. This method uses the approximate power iteration [10] to obtain a basis update instead of computing the shift parameters of the ADI iteration. It has been shown that when $B$ is a vector, the parameter free algorithm is equivalent to the $\mathrm{ADI}$ method if the shift parameters are identical to the 
eigenvalues of the projected matrix. Therefore, the parameter free method is also an ADI method, and its shift parameters are automatically chosen via solving a standard Sylvester equation.

The parameter free iterative method presented in this paper for solving the projected generalized Lyapunov equation (1) is an extension of the work in [9]. The algorithm is developed based upon a synthesis of the approximate power method and the generalized low-rank ADI method [6]. We show that when $B$ is a vector, the parameter free method for projected generalized Lyapunov equations generates the same updates as the generalized low-rank ADI method with shift parameters being the eigenvalues of a projected matrix. Moreover, the performance of the newly proposed method is compared to that of the generalized low-rank ADI method.

Throughout this paper, we adopt the following notations. We denote by $I$ the identity matrix, and by 0 the zero vector or zero matrix. The dimensions of these vectors and matrices, if not specified, are deduced by the context. The Frobenius matrix norm is denoted by $\|\cdot\|_{F}$. The superscript ".$T "$ stands for the transpose only.

The remainder of the paper is organized as follows. In Section II, we propose a parameter free iterative method for solving the projected Lyapunov equation. Section III is devoted to some numerical tests. Conclusions are given in the last section.

\section{A parameter free iterative method}

We always assume that the pencil $\lambda E-A$ is c-stable, i.e., all their finite eigenvalues have negative real part. Thus, the projected generalized continuous-time Lyapunov equation (1) has a unique, symmetric and positive semidefinite solution. It follows from the assumption that $A$ is nonsingular, hence the projected generalized Lyapunov equation (1) is equivalent to the projected standard Lyapunov equation

$$
\left\{\begin{array}{l}
\left(A^{-1} E\right) X+X\left(A^{-1} E\right)^{T}+P_{r} A^{-1} B B^{T} A^{-T} P_{r}^{T}=0 \\
X=P_{r} X P_{r}^{T}
\end{array}\right.
$$

In [9], Nong and Sorensen proposed a parameter free method for solving the large-scale Lyapunov equation in low-rank factored form. The algorithm is based upon a synthesis of the approximate power method and the alternating direction implicit method. In this section, we will generalize this method for solving Equation (3). The algorithm is described as follows.

\section{Algorithm 1. A parameter free iterative method}

Input: $A, E \in R^{n \times n}$ and $B \in R^{n \times m}$ with $\lambda E-A$ being c-stable.

Output: $Z_{j}$ such that $X_{j}=Z_{j} Z_{j}^{T}$ is an approximate solution of Equation (3).

1. Let $A=A^{-1} E, B_{1}=P_{r} A^{-1} B$ and $Z_{1}=0$. Compute $U_{1}=\operatorname{orth}\left(B_{1}\right)$.

2. For $j=1,2, \cdots$

- Set $H_{j}=U_{j}^{T} A U_{j}$ and $B_{j}=U_{j}^{T} B_{j}$.

- Solve the following low-dimensional Lyapunov equation for $R_{j}$

$$
H_{j} R_{j}+R_{j} H_{j}^{T}+B_{j} B_{j}^{T}=0
$$


- Solve the projected Sylvester equation for $Y_{j}$

$$
\left\{\begin{array}{l}
A Y_{j}+Y_{j} H_{j}^{T}+B_{j} B_{j}^{T}=0, \\
Y_{j}=P_{r} Y_{j} .
\end{array}\right.
$$

- Compute $Y_{j}=Y_{j} R_{j}^{-1 / 2}$. The matrix $Y_{j} Y_{j}^{T}$ is an approximate solution of the updated equation

$$
\left\{\begin{array}{l}
A_{X_{j}}+X_{j} A^{T}+B_{j} B_{j}^{T}=0 \\
X_{j}=P_{r} X_{j} P_{r}^{T}
\end{array}\right.
$$

- Compute the short singular value decomposition of the matrix $\left[Z_{j}, Y_{j}\right]$

$$
[U, \Sigma, V]=\operatorname{svd}\left(\left[Z_{j}, Y_{j}\right], 0\right)
$$

where the diagonal entries of $\Sigma$ are ordered decreasingly.

- Let $Z_{j+1}=U \Sigma$ and $U_{j+1}=U\left(:, 1: k_{j}\right)$ If the approximate solution $X_{j+1}=Z_{j+1} Z_{j+1}^{T}$ is accurate enough, then stop.

Set $B_{j+1}=\left(I-Y_{j} R_{j}^{-1} U_{j}^{T}\right) B_{j}$.

About Algorithm 1, some remarks of implementation details are in order:

The product of $A^{-1}$ with some matrix should be implemented by solving the linear systems of equations with the coefficient matrix $A$ and multiple right-hand sides. To do it, the $L U$ factorization [11] of $A$ is employed for medium-size matrices, and the Cholesky factorization of $A$ should be used for $A$ symmetric positive definite. For large-scale matrices, a preconditioning iterative method could be employed to solve systems with $A$, where the preconditioner could be generated once for all. Iterative methods that are used nowadays are Krylov subspace methods such as GMRES [12].

The function $\operatorname{orth}\left(B_{1}\right)$ stands for the modified Gram-Schmidt process [11] for generating an orthonormal basis for the range of $B_{1}$.

Note that $R_{j}$ is obtained from the Lyapunov equation $H_{j} k R_{j}+R_{j} H_{j}^{T}+B_{j} B_{j}^{T}=0$. We assume that $A=A^{-1} E$ is dissipative on its projection subspace corresponding to its non-zero eigenvalues. Thus, $H_{j}=U_{j}^{T} A U_{j}$ is stable and this Lyapunov equation admits a unique solution $R_{j}$. In the following discussion, it is assumed that $R_{j}$ is always positive definite. 
At each iterative step, we need to solve the projected Sylvester equation (4) for $Y_{j}$. This special Sylvester equation can be solved efficiently by the method proposed in [13].

The following theorem shows that if $B$ is a vector, the parameter free iterative method is an ADI-type method.

Theorem 1. Let $A=A^{-1} E$ and $H_{j}, B_{j}, B_{j}$ be defined as in Algorithm 1. Assume that $B$ is a vector, the pencil $\lambda E-A$ is c-stable, $H_{j}$ is stable, and $\left(H_{j}, B_{j}\right)$ is controllable. Then the update $Y_{j} R_{j}^{-1} Y_{j}^{T}$ at Step $j$ in Algorithm 1 is precisely the same as the approximate solution obtained by applying the generalized low-rank ADI method with shift parameters $\left\{\mu_{i}\right\}_{i=1}^{k}$ being the eigenvalues of the matrix $H_{j}$ to

$$
\left\{\begin{array}{l}
A_{X_{j}}+X_{j} A^{T}+B_{j} B_{j}^{T}=0 \\
X_{j}=P_{r} X_{j} P_{r}^{T}
\end{array}\right.
$$

Proof. Let $\left\{\mu_{i}\right\}_{i=1}^{k}$ being the eigenvalues of the matrix $H_{j}$. For $i=1,2, \cdots, k$, define

$$
\begin{aligned}
A_{\mu_{i}} & =\left(A-\bar{\mu}_{i} I\right)\left(A+\mu_{i} I\right)^{-1} \\
& =\left(A^{-1} E-\bar{\mu}_{i} I\right)\left(A^{-1} E+\mu_{i} I\right)^{-1}, \\
H_{\mu_{i}} & =\left(H_{j}-\bar{\mu}_{i} I\right)\left(H_{j}+\mu_{i} I\right)^{-1}, \\
B^{(i)} & =\sqrt{-2 \operatorname{Re}\left(\mu_{i}\right)}\left(A+\mu_{i} I\right)^{-1}\left(\prod_{s=1}^{i-1} A_{\mu_{i}}\right) B_{j}, \\
B^{(i)} & =\sqrt{-2 \operatorname{Re}\left(\mu_{i}\right)}\left(H_{j}+\mu_{i} I\right)^{-1}\left(\prod_{s=1}^{i-1} H_{\mu_{i}}\right) B_{j} .
\end{aligned}
$$

Applying the generalized low-rank ADI method with shift parameters $\left\{\mu_{i}\right\}_{i=1}^{k}$ to the updated equation (5), we obtain an approximate solution $L_{j} L_{j}^{*}$ of (5), where

$$
L_{j}=\left[B^{(1)}, B^{(2)}, \cdots, B^{(k)}\right]
$$

It is easy to verify that

$$
\begin{aligned}
P_{r}\left(A^{-1} E-\bar{\mu}_{i} I\right) & =\left(A^{-1} E-\bar{\mu}_{i} I\right) P_{r}, \\
P_{r}\left(A^{-1} E+\mu_{i} I\right)^{-1} & =\left(A^{-1} E+\mu_{i} I\right)^{-1} P_{r},
\end{aligned}
$$


which together with $B_{j}=P_{r} B_{j}$ shows $B^{(i)}=P_{r} B^{(i)}$. Then we immediately get $L_{j} L_{j}^{*}=P_{r} L_{j} L_{j}^{*} P_{r}^{T}$, i.e., the second equation in (5) is satisfied exactly by $L_{j} L_{j}^{*}$.

Let

$$
L_{j}=\left[B^{(1)}, B^{(2)}, \cdots, B^{(k)}\right]
$$

The solution $Y_{j}$ of

$$
\left\{\begin{array}{l}
A Y_{j}+Y_{j} H_{j}^{T}+B_{j} B_{j}^{T}=0 \\
Y_{j}=P_{r} Y_{j}
\end{array}\right.
$$

can be expressed as $Y_{j}=L_{j} L_{j}^{T}$, and the solution $R_{j}$ of the equation

$$
H_{j} R_{j}+R_{j} H_{j}^{T}+B_{j} B_{j}^{T}=0
$$

can be formulated as $R_{j}=L_{j} L_{j}^{T}$.

Since $B$ is a vector and $\left(H_{j}, B_{j}\right)$ is controllable, $L_{j}$ is invertible. Hence

$$
Y_{j} R_{j}^{-1} Y_{j}^{T}=L_{j} L_{j}^{T}\left(L_{j} L_{j}^{T}\right)^{-1} L_{j} L_{j}^{T}=L_{j} L_{j}^{T}
$$

\section{Numerical experiments}

In this section, we present two numerical examples to illustrate the performance of the parameter free iterative method (Algorithm 1) for the projected generalized Lyapunov equation (1). Algorithm 1 is denoted by PFIM. For the purpose of comparison, we also present the test results obtained by the generalized low-rank alternating direction implicit method (denoted by LR-ADI) proposed in [6]. In the following examples, we compare the numerical behavior of the two methods with respect to the number of iterations (ITs), CPU time (in seconds) and the relative residuals (RES). Here the relative residuals are defined by

$$
R E S=\frac{\left\|E X_{j} A^{T}+A X_{j} E^{T}+P_{l} B B^{T} P_{l}^{T}\right\|_{F}}{\left\|P_{l} B B^{T} P_{l}^{T}\right\|_{F}},
$$

where $X_{j}$ denotes the $j$ th iterate of PFIM or LR-ADI. The stopping criterion for both methods is $R E S<10^{-12}$. 
All numerical experiments are performed on an Intel Pentium Dual E2160 with CPU $1.80 \mathrm{GHz}$ and RAM 1GB under the Window XP operating system and the usual double precision, where the floating point relative accuracy $2.22 \times 10^{-16}$.

\subsection{Example 1}

For the first experiment, we consider the 2D instationary Stokes equation that describes the flow of an incompressible fluid in a domain. The spatial discretization of this equation by the finite difference method on a uniform staggered grid leads to the descriptor system

$$
\begin{aligned}
E \dot{x}(t) & =A x(t)+B u(t), \\
y(t) & =C x(t) .
\end{aligned}
$$

This example for the projected generalized Lyapunov equations was presented by Stykel, see [6] and the references therein. The matrix coefficients in (6) are given by

$$
E=\left[\begin{array}{cc}
E_{11} & 0 \\
0 & 0
\end{array}\right] \in R^{n \times n}, \quad A=\left[\begin{array}{cc}
A_{11} & A_{12} \\
A_{21} & 0
\end{array}\right] \in R^{n \times n} .
$$

If $E_{11}$ and $A_{21} E_{11}^{-1} A_{12}$ are nonsingular, then the pencil $\lambda E-A$ is of index 2 . These matrices are sparse and have special block structure. Using this structure, the projectors $P_{l}$ and $P_{r}$ onto the left and right deflating subspaces of the pencil $\lambda E-A$ can be computed as

$$
\begin{aligned}
& P_{l}=\left[\begin{array}{ccc}
\prod_{l} & -\prod_{l} A_{11} E_{11}^{-1} A_{12}\left(A_{21} E_{11}^{-1} A_{12}\right)^{-1} \\
0 & 0 &
\end{array}\right], \\
& P_{r}=\left[\begin{array}{cc}
\Pi_{r} & 0 \\
-\left(A_{21} E_{11}^{-1} A_{12}\right)^{-1} A_{21} E_{11}^{-1} A_{11} \Pi_{r} & 0
\end{array}\right],
\end{aligned}
$$

where $\Pi_{l}=I-A_{12}\left(A_{21} E_{11}^{-1} A_{12}\right)^{-1} A_{21} E_{11}^{-1}$ is a projector onto the kernel of $A_{21} E_{11}^{-1}$ along the image of $A_{12}$ and $\Pi_{r}=I-E_{11}^{-1} A_{12}\left(A_{21} E_{11}^{-1} A_{12}\right)^{-1} A_{21}=E_{11}^{-1} \Pi_{l} E_{11}$. In this example, the state space dimensions of the problem are $n=1280$ and $m=1$.

The results in Table I show that the PFIM method needs 8 steps of iterations and 0.58 seconds for reaching the relative residual $2.8 \mathrm{e}-014$ while the LR-ADI method 18 iterations and 4.38 seconds for convergence. It clearly indicates that the PFIM method is more efficient than the LR-ADI method for this example. 
Table 1.

\begin{tabular}{|c|c|c|c|}
\hline & ITs & CPU & RES \\
\hline LR-ADI & 18 & 4.38 & $1.7 \times 10^{-13}$ \\
\hline PFIM & 8 & 0.58 & $2.8 \times 10^{-14}$ \\
\hline
\end{tabular}

Here and in the following, the LR-ADI method uses the heuristic algorithm proposed by Penzl [7] to compute the suboptimal shift parameters. This algorithm is based on Arnoldi iterations [13] applied to the matrices $A^{-1} E$ and $P A$ with $P=P_{r}\left(E P_{r}-A Q_{r}\right)^{-1}$, see [6] for the details.

\subsection{Example 2}

We now do the same experiment as in the previous example except that $n$ is 3007 instead of 1280 .

From Table II, we can see that for $n=3007$, the number of iterations is almost the same as that for $n=1280$. The PFIM method costs 1.57 seconds for convergence while the LR-ADI method needs 13.8 seconds.

Table 2.

\begin{tabular}{|c|c|c|c|}
\hline & ITs & CPU & RES \\
\hline LR-ADI & 20 & 13.8 & $4.3 \times 10^{-13}$ \\
\hline PFIM & 8 & 1.57 & $9.2 \times 10^{-14}$ \\
\hline
\end{tabular}

\section{Conclusions}

In this paper, we have proposed a parameter free iterative method to solve the projected continuous-time generalized Lyapunov equation. The new method is developed based upon a combination of an approximate power method and a low-rank ADI method. Numerical experiments are presented for the performance comparison between the parameter free iterative method and the generalized low-rank ADI method. It shows that the method proposed in this paper outperforms the low-rank ADI method.

\section{References}

[1] T. Stykel, "Stability and inertia theorems for generalized Lyapunov equations," Linear Algebra Appl., vol. 355, pp. 297-314, 2002.

[2] F. Gantmacher, Theory of Matrices. New York: Chelsea, 1959.

[3] B. Anderson and J. Moore, Optimal Control-Linear Quadratic Methods. Englewood Cliffs, NJ: PrenticeHall, 1990.

[4] V. Mehrmann, The Autonomous Linear Quadratic Control Problem, Theory and Numerical Solution, ser. Lecture Notes in Control and Information Sciences. Heidelberg: Springer, 1991, vol. 163.

[5] T. Stykel, "Numerical solution and perturbation theory for generalized Lyapunov equations," Linear Algebra Appl., vol. 349, pp. 155-185, 2002.

[6] T. Stykel, "Low-rank iterative methods for projected generalized Lyapunov equations," Elect. Trans. Numer. Anal., vol. 30, pp. 187-202, 2008. 
[7] T. Penzl, "A cyclic low-rank Smith method for large sparse Lyapunov equations," SIAM J. Sci. Comput., vol. 21, pp. 1401-1418, 1999/2000.

[8] P. Benner, H. Mena, and J. Saak, "On the parameter selection problem in the Newton-ADI iteration for large-scale Riccati equations,” Elect. Trans. Numer. Anal., vol. 29, pp. 136-149, 2008.

[9] R. Nong and D. C. Sorensen, "A parameter free adi-like method for the numerical solution of large scale lyapunov equations," 2009, preprint.

[10] A. Hodel, B. Tenison, and K. Poolla, "Numerical solution of the Lyapunov equation by approximate power iteration," Linear Algebra Appl., vol. 236, pp. 205-230, 1996.

[11] G. H. Golub and C. F. V. Loan, Matrix Computations, 3rd ed. Baltimore, MD: John Hopkins University Press, 1996.

[12] Y. Saad and M. H. Schultz, "GMRES: A generalized minimal residual algorithm for solving nonsymmetric linear systems,” SIAM J. Sci. Statist. Comput., vol. 7, pp. 856-869, 1986.

[13] D. Vasilyev and J. White, "A more reliable reduction algorithm for behavior model extraction," in Proc. Int. Conf. on Computer. Aided Design (ICCAD 2005), 2005, pp. 812-819. 